\title{
Cancer de próstata órgano-confinado: Tratamiento con altas dosis de radioterapia (radioterapia de intensidad modulada)
}

\author{
Livi L*, Detti B*, Meattini M*, Sánchez LJ**, Biti GP* \\ *Departamento de Radioterapia. Universidad de Florencia. ${ }^{* *}$ AOUC SOD ${ }^{* * *}$ Chirugica. Italia.
}

Actas Urol Esp. 2007;31(6):611-616

\section{RESUMEN}

\section{CANCER DE PRÓSTATA ÓRGANO-CONFINADO: TRATAMIENTO CON ALTAS DOSIS DE RADIOTERAPIA} (RADIOTERAPIA DE INTENSIDAD MODULADA)

Objetivo: Determinar la toxicidad local así como la supervivencia de los pacientes con cáncer de próstata tratados con altas dosis de radioterapia.

Material y métodos: Entre junio de 2003 y mayo de 2006100 pacientes fueron sometidos a tratamiento radioterápico por cáncer de prostáta localizado. Se administró una dosis de $80 \mathrm{~Gy}$ al target con una técnica de dos fases (CRT+IMRT). El parámetro medio de PSA fue de 9. La media del seguimiento ha sido de 12 meses.

Resultados: Dieciocho (18\%) de los pacientes desarrollaron toxicidad GI aguda grado 2 y ningún tipo de toxicidad grado 3 o mayor. El $44 \%$ de los paciente experimentaron toxicidad GU grado 2 mientras que el $34 \%$ de ellos no experimentaron síntomas GU durante el tratamiento. El 3\% de los pacientes desarrollaron toxicidad GI grado 2 y el $8 \%$ toxicidad GU tardías. Ningun paciente experimentó síntomas mas severos.

Se registró recaída bioquímica en dos pacientes. Ambos tenían factores pronósticos de alto riesgo en el momento del diagnóstico de cáncer de próstata.

Conclusión: Los datos demuestran la seguridad y eficacia de las altas dosis de radioterapia en los pacientes con cáncer de próstata localizado utilizando una escalada de dosis en el tratamiento con bajos niveles de toxicidad en tejidos normales.

Palabras clave: Radioterapia de intensidad modulada. Cáncer de próstata. Toxicidad rectal. Radioterapia.

\section{ABSTRACT \\ ORGAN-CONFINED PROSTATE CANCER: TREATMENT WITH HIGH DOSES OF RADIOTERAPY. (INTENSITY MODULATED RADIOTHERAPY)}

Purpose: To report toxicity and local control in patients with localized prostate cancer, treated with high dose radiotherapy.

Materials and methods: The records of 100 consecutive patients with clinically localized prostate

cancer treated between june 2003 and may 2006 were reviewed. They received 80 Gy to the target volume with a biphasic technique (3DCRT + IMRT). The median pretreatment PSA was 9. The median follow-up time was 12 months.

Results: Eighteen (18\%) developed acute Grade 2 rectal toxicity, and no patient experienced acute grade 3 or higher rectal symptoms. Forty-four (44\%) developed acute Grade 2 urinary symptoms while $34 \%$ of the patients experienced no GU symptoms (Grade 0) during treatment. Three patients (3\%) developed late rectal toxicity grade 2 and eight patients ( $8 \%$ ) experienced late urinary toxicity grade 2 ; any patients experienced more severe symptoms.

We recorded biochemical relapse in two patients, both had poor prognostic factors at initial diagnosis of prostate cancer.

Conclusions: The data demonstrate the feasibility and safety of high dose radiotherapy for patients with localized prostate cancer and provide a proof that this method allow safe dose escalation with low severe toxicities to the normal tissues.

Keywords: Intensity modulated radiotherapy treatment. Prostate cancer. Rectal toxicity. Radiotherapy. 
$\mathrm{E}^{1}$ desarrollo y el empleo de nuevas técnicas, como la radioterapia conformacional 3D (3DCRT) y la radioterapia de intensidad modulada (IMRT) han permitido tratar el cáncer prostático con dosis más altas de radiación en comparación con las técnicas de radioterapia convencional pero sin incrementar la toxicidad en los tejidos sanos circundantes ${ }^{1-4}$.

La "escalada de dosis" es, en efecto, una de las estrategias más eficaces para mejorar el control local y la supervivencia de los pacientes con cáncer de próstata.

Además, datos recientes sugieren que la mejoría del control local de la enfermedad determina, también, una mejor supervivencia libre de metástasis ${ }^{1}$.

Sin embargo, un aumento de la dosis de radiaciones, puede comportar también el riesgo de desarrollar complicaciones, secundarias a la afectación actínica de la vejiga y el recto. Muchos estudios han demostrado que la incidencia de complicaciones rectales y genitourinarias (GU) no está asociada, exclusivamente, con la dosis de radiación en el órgano afecto sino también con el volumen total irradiado ${ }^{5-6}$.

Recientemente, se ha observado que un aumento de la dosis al target de 64,8 a $81 \mathrm{~Gy}$, con las técnicas convencionales 3D-CRT, implica una incidencia más alta de toxicidad rectal y urinaria de Grado 2. La incidencia de sangrado rectal crónico de Grado 2 entre los pacientes que han recibido 75.6 - 81 Gy ha sido del 17\% versus el 6\% en aquellos tratados con una dosis menor ${ }^{2-4,7}$. Storey ha demostrado que pacientes con $>25 \%$ de volumen rectal irradiado con 70 Gy con la técnica 3DCRT tienen un riesgo significativamente mayor de desarrollar complicaciones de Grado $\geq 2$, y además, que la sintomatología de Grado 3 se manifiesta cuando han recibido irradiación sobre $>30 \%$ del recto y más de $\geq 70$ Gy en total ${ }^{8}$.

Kutcher y colaboradores han estimado que manteniendo una dosis de 75 Gy al 30\% del recto, el riesgo de toxicidad tardía del aparato gastrointestinal es inferior al 10\% ${ }^{9}$.

\section{MATERIALES Y MÉTODOS}

Entre junio de 2003 y mayo de 2006, en nuestro Instituto de Radioterapia 100 pacientes han sido sometidos a tratamiento radioterápico por cáncer de prostáta localizado.
Todos los pacientes tenían confirmación histológica de adenocarcinoma y una determinación de PSA antes del tratamiento. El estudio de extensión (TAC abdomino-pélvico, gammgrafía ósea) fue negativo para metástasis.

La edad media de los pacientes fue de 68 años (rango: 55-82). Según la clasificación TNM 10 pacientes pertenecían al estadio clínico T1, 25 al estadio T2 y los 65 restantes al estadio T3. El PSA medio antes del tratamiento fue de 9, respectivamente (rango de 3-53). La suma de Gleason estuvo comprendida entre 4 y 9 (40 pacientes con Gleason $\leq 6$, 30 con 7 y los 30 restantes con $\geq 8$ ). Sesenta pacientes recibieron bloqueo androgénico durante y/o después de la radioterapia. Una dosis de 80 Gy fue administrada al target (2 Gy de fracción diaria para un total de 40 aplicaciones). Todos los pacientes han sido visitados semanalmente para evaluar el grado de toxicidad debido al tratamiento.

Después del tratamiento radioterápico, los pacientes han sido sometidos a seguimiento clínico a los 3 meses y posteriormente cada 4 meses. En cada una de estas visitas se ha realizado tacto rectal y PSA (Tabla 1).

La media del seguimiento ha sido de 12 meses (rango 3-35 meses).

Tabla 1. Correlación entre estadio y suma de Gleason.

\begin{tabular}{lccc}
\hline $\begin{array}{l}\text { Intracapsular } \\
\text { (35 pacientes) }\end{array}$ & Gleason $\geq \mathbf{6}$ & Gleason $=\mathbf{7}$ & Gleason $\geq \mathbf{8}$ \\
\hline T1 & 10 & 0 & 0 \\
T2 & 5 & 10 & 10 \\
\hline Extracapsular & & & \\
(65 pacientes) & Gleason $\leq \mathbf{6}$ & Gleason $=\mathbf{7}$ & Gleason $\geq \mathbf{8}$ \\
\hline T3a & 15 & 10 & 10 \\
T3b & 10 & 10 & 10 \\
\hline
\end{tabular}

\section{TRATAMIENTO}

El tratamiento se ha realizado con una técnica de dos fases (40 Gy con técnica 3D-CRT y 40 Gy con técnica IMRT).

La primera fase ha consistido en el tratamiento estándar con cuatro campos conformacionales (angulos gantry 0, 90, 180, 270).

En la segunda fase se ha adoptado una técnica IMRT a cinco campos, (ángulos gantry 45, 
105, 180, 255, 315), usando un algoritmo de planning inverso para conseguir perfiles de intensidad optimizados para el tratamiento diseñado.

Los haces a intensidades moduladas han sido distribuidos con técnica MLC segmentada (sMLC), conocidas también como técnica step and shoot.

Hemos decidido utilizar una técnica mixta por razones prácticas y teóricas: la técnica 3D-CRT es más simple de planificar y el tiempo necesario para administrar cada fracción es más breve con respecto a la técnica IMRT. Sin embargo, ésta última nos permite administrar altas dosis al target, con una mejoría del control local y un bajo riesgo de complicaciones rectales y genitourinarias.

Todos los tratamientos han sido planificados usando el sistema Xio, (CMS, Saint Louis). El tratamiento se ha realizado con nuestro sistema ELEKTA Precise Linacs. Para la planificación del tratamiento, los datos volumétricos del TC se han conseguido con el paciente en posición supina. Se han colocado marcadores radio-opácos sobre los tatuajes para evidenciar los puntos de triangulación del isocentro a la TC. El volumen clínico del target (CTV) ha incluido sólo la próstata en 40 pacientes, mientras en 60 pacientes el CTV ha incluido la próstata y las vesículas seminales, porque estaban radiológicamente implicadas o por el riesgo de una afectación seminal superior al 15\%, de acuerdo con los tablas de Partin ${ }^{10}$. Un margen de $0.8 \mathrm{~cm}$ ha sido añadido alrededor del CTV para definir el PTV, excepto en la parte posterior, entre la pared rectal anterior y la próstata misma, dónde se ha utilizado un margen de $0,5 \mathrm{~cm}^{11}$ para reducir el riesgo de toxicidad rectal.

Para el recto y la vejiga han sido utilizados los siguientes vínculos de dosis:

1. Porcentaje de volumen rectal irradiado con más de 65 Gy <30\%;

2. Porcentaje de volumen rectal irradiado con más de 70 Gy <25\%;

3. Porcentaje de volumen rectal irradiado con más de 75 Gy <10\%;

4. Porcentaje de volumen rectal irradiado con más de 78 Gy $<2 \%$

5. NTCP sujetado <10\%;

6. NTCP vejiga $<1 \%$, entreambos los NTCP han sido calculados con el método de Lyman Kutcher - Burman y los datos radiobiológicos llevados por Emami y Goiten ${ }^{12-15}$.
Nuestro procedimiento, que asegura el control de calidad para el IMRT consiste en el protocolo siguiente: antes del TAC de planificación los pacientes tienen que efectuar un enema de modo que simulen las peores condiciones en el cálculo de riesgo de toxicidad rectal. Se necesita además, que los pacientes antes de la TAC de centrado y del tratamiento radioterápico, se presenten con la vejiga llena.

Durante la TAC de centrado y del tratamiento, todos los pacientes deben estar en posición supina con los miembros inferiores inmovilizados con cinturones adecuados, los brazos cruzados sobre el tórax y la cabeza apoyada sobre de una almohada.

La dosis que damos con la CTV ha sido de 80 Gy; la dosis mínima solicitada para la cobertura del PTV ha sido de 74 Gy $(92.5 \%$ de la dosis al CTV). Siguiendo las guías establecidas por la ICRU, la cobertura ha sido aceptada como óptima si el 95\% de la isodosis cubrió todas las estructuras $^{16-20}$

Todos los set-up de tratamiento han sido establecidos mediante el soporte de imágenes portales (Elekta I view amorphous silicon portal imaging system) utilizando tres angulos gantry $\left(0^{\circ}\right.$, $90^{\circ} \mathrm{e} 45^{\circ}$ ). En el caso de errores de set-up superiores a los $3 \mathrm{~mm}$, las correcciones han sido efectuadas antes del tratamiento.

La toxicidad aguda ha sido registrada una vez a la semana en el curso del tratamiento, usando la clasificación de toxicidad EORTC/RTOG y los criterios comunes de toxicidad (CTC version 3.0).

La toxicidad tardía (6 meses después del tratamiento) ha sido evaluada de acuerdo con la escala de la Radiation Therapy Oncology Group ${ }^{21}$.

\section{RESULTADOS}

\section{Toxicidad aguda}

En general, el 82\% de los pacientes no ha sufrido molestias gastrointestinales (GI) o ellas fueron mínimas (Grado 0/1); mientras, en el $18 \%$ restante se ha obtenido una toxicidad GI aguda Grado 2 .

Globalmente, el 34\% de los pacientes no ha informado sintomas GU, grado 0, durante el tratamiento, el $22 \%$ ha desarrollado toxicidad urinaria de Grado 1 y el 44\% ha evidenciado un Grado 2 como máximo nivel de toxicidad aguda vesical (Tablas 2 y 3 ). 
Tabla 2. Toxicidad gastrointestinal y genito-urinaria agudas.

\begin{tabular}{lcc}
\hline & Gastrointestinal & Genitourinaria \\
\hline Grado 0 & 50 pts (50\%) & 34 pts (34\%) \\
Grado 1 & 32 pts (32\%) & 22 pts (22\%) \\
Grado 2 & 18 pts (18\%) & 44 pts (44\%) \\
Grado 3 & - & - \\
\hline
\end{tabular}

Tabla 3. Toxicidad gastrointestinal y genitourinaria tardía.

\begin{tabular}{lcc}
\hline & Gastrointestinal & Genitourinaria \\
\hline Grado 0 & $88 \mathrm{pts}(88 \%)$ & $82 \mathrm{pts} \mathrm{(82 \% )}$ \\
Grado 1 & $9 \mathrm{pts}(9 \%)$ & $10 \mathrm{pts} \mathrm{(10 \% )}$ \\
Grado 2 & $3 \mathrm{pts} \mathrm{(3 \% )}$ & $8 \mathrm{pts} \mathrm{(8 \% )}$ \\
Grado 3 & - & - \\
\hline
\end{tabular}

\section{Toxicidad tardia}

En la última revisión del seguimiento hemos hallado 3 pacientes (3\%) con toxicidad GI tardía de Grado 2 y 8 pacientes (8\%) con toxicidad tardía GU de Grado 2. Ningún paciente ha manifestado sintomas más severos.

\section{Resultados en términos de control de la enfermedad}

Hemos registrado recaida bioquímica en dos pacientes. Ambos tuvieron factores pronósticos de alto riesgo como un PSA pretratamiento $>20$ $\mathrm{ng} / \mathrm{ml}$, una suma de Gleason $>8$ y un estadio clínico T3b. Han sido sometidos a bloqueo androgénico total después del diagnóstico de recaida bioquímica.

\section{DISCUSIÓN}

Con respecto a los tratamientos convencionales, las técnicas 3D-CRT e IMRT permiten administrar una dosis de irradiación mayor sobre la próstata, con preservación de los los tejidos sanos. Eso ha permitido un mejor control local de la enfermedad y una buena tolerancia por los órganos en riesgo, que rodean el objetivo central.

Pollack ha demostrado que un incremento de la dosis de $8 \mathrm{~Gy}$, de 70 a $78 \mathrm{~Gy}$, ha llevado a una mejoría de la supervivencia libre de recidiva del $69 \%$ al $79 \% 4$.

Hanks y colaboradores han demostrado que el tiempo hasta la recidiva y el tiempo de supervivencia fueron mayores para los pacientes trata- dos con dosis $\geq 74 \mathrm{~Gy}$, con respecto a los pacientes tratados con dosis menores de 74 Gy ${ }^{22}$.

Zelefsky ha reportado un riesgo de toxicidad rectal de grado 2 del 16\% en los pacientes tratados con técnica 3D-CRT a dosis de 75.6-81 Gy y efectos colaterales tardíos GU en el 15\% de los pacientes tratados ${ }^{23}$.

Peeters et al. ${ }^{14}$ han demostrado que aumentando la dosis de 68 Gy a 78 Gy se observa un aumento de la incidencia de sangrado rectal y un mayor porcentaje de nocturia tardía. Además, han informado cómo los pacientes sometidos a hormonoterapia han manifestado una mayor toxicidad tardía, probablemente ligada al efecto inhibitorio de las hormonas sobre los procesos de reparación de los tejidos normales irradiados.

En el 2000 Zelefsky $^{7}$ ha confrontado la incidencia de la toxicidad aguda y tardía en los pacientes tratados con técnica IMRT, con respecto de aquellos tratados con 3D-CRT a altas dosis. Ha encontrado un riesgo actuarial a 2 años de sangrado rectal del $2 \%$ en los pacientes tratados con técnico IMRT y del 10\% en aquellos tratados con la técnica 3DCRT ( $\mathrm{p}<0,001$ ).

Zelefsky et al. ${ }^{2}$ han informado que las complicaciones rectales de Grado 2, a 3 años, han sido del 14\% en los pacientes tratados a una dosis de 81 Gy con técnica 3D-CRT y del 2\% en aquellos tratados con IMRT.

El mismo grupo ha publicado en 2002, los datos sobre 772 pacientes tratados con IMRT (698 tratados con una dosis de 81 Gy y 74 con una dosis de 86 Gy) ${ }^{25}$; las toxicidades tardias de Grado 2 GI y GU han sido del 1,5\% y del 9\%, respectivamente.

De Meerleer en el $2004^{26}$ ha publicado datos sobre toxicidad aguda en 114 pacientes con cáncer de próstata tratado con técnico IMRT. Se ha informado toxicidad GI de grado 1 y grado 2 en el $44 \%$ y en el 29\% de los pacientes, respectivamente. Además, observó toxicidad GU de grado 1 en el $47 \%$ de los pacientes, de grado 2 en el $26 \%$ y de grado 3 en el $7 \%$.

Kupelian et al. ${ }^{27}$ han observado efectos colaterales durante el tratamiento con técnica IMRT con menor fraccionamiento en 166 pacientes. La toxicidad rectal aguda de Grado 1 y Grado 2 se informó en el 55\% y en el 15\% de los pacientes. 
Recientemente, Zelefsky ha publicado datos sobre 561 pacientes tratados con IMRT con dosis de 81 Gy. Sólo en 3 pacientes hubo toxicidad rectal tardía de grado 3 y en 7 pacientes sangrado rectal de grado $2^{28}$.

\section{CONCLUSIONES}

La técnica IMRT representa una de las principales novedades en la tecnologia radioterápica, que nos permite utilizar una escalada de dosis en el tratamiento del tumor prostático.

En nuestra experiencia la radioterapia standard con técnica 3DCRT e IMRT, ha resultado segura y eficaz. A pesar de las altas dosis suministradas no hemos registrado toxicidad grave en los órganos sanos circundantes.

Aunque nuestro análisis resulte limitado por el reducido número de pacientes, somos de la opinión que el empleo de altas dosis de radioterapia en el tratamiento del tumor prostático, nos ha dado óptimos resultados en términos de control de enfermedad y efectos colaterales, agudos y tardios.

\section{REFERENCIAS}

1. Coen JJ, Zietman AL, Thakral H, Shipley WU. Radical radiation for localized prostate cancer: local persistence of disease results in a late wave of metastases. J Clin Oncol. 2002 Aug 1;20(15):3199-3205.

2. Zelefsky MJ, Leibel SA, Gaudin PB, Kutcher GJ, Fleshner NE, Venkatramen ES et al. Dose escalation with three dimensional conformal radiation therapy affects the outcome in prostate cancer. Int J Radiat Oncol Biol Phys. 1998 Jun 1;41(3):491-500.

3. Hanks GE, Hanlon AL, Pinover WH, Horwitz EM, Price RA, Schultheiss T. Dose selection for prostate cancer based on dose comparison and dose response studies. Int $J$ Radiat Oncol Biol Phys. 2000 Mar 1;46(4):823-832.

4. Pollack A, Zagars GK, Smith LG, et al. Preliminary results of a randomized radiotherapy dose escalation study comparing 70 Gy to 78 Gy for prostate cancer. J Clin Oncol. 2000 Dec 1;18(23):3904-3911.

5. Sandler HM, McLaughlin W, Kish KE, Lichter AS. Results of 3D conformal radiotherapy in the treatment of 707 patients with localized prostate cancer. Int $J$ Radiat Oncol Biol Phys. 1995;32:141.

6. Lee, WR, Hanks GE, Hanlon AL, SchultheissTE, Hunt MA. Lateral rectal shielding reduces late rectal morbidity following high dose three-dimensional conformal radiation therapy for clinically localized prostate cancer: further evidence for a significant dose effect. Int J Radiat Oncol Biol Phys. 1996 May 1;35(2):251-257.

7. Zelefsky MJ, Fuks Z, Hunt M, Lee HJ, Lombardi D, Ling CC, et al. High dose radiation delivered by intensity modulated conformal radiotherapy improves the outcome of localized prostate cancer. J Urol. 2001 Sep;166(3):876-881.
8. Storey MR, Pollack A, Zagars G, Smith L, Antolak J, Rosen I. Complications from radiotherapy dose escalation in prostate cancer: preliminary results of a randomized trial. Int J Radiat Oncol Biol Phys. 2000 Oct 1;48(3):635-642.

9. Kutcher GJ, Leibel SA, Ling CC, Zelefsky M, Fuks Z. New wine in an old bottle? Dose escalation under dose-volume constraints: a model of conformal therapy of the prostate. Int J Radiat Oncol Biol Phys. 1996 May 1;35(2):415-416.

10. Partin AW, Yoo J, Carter HB, Pearson JD, Chan DW, Epstein JI, Walsh PC. The use of prostate specific antigen, clinical stage, and Gleason score to predict pathological stage in men with localized prostate cancer. J Urol. 1993 Jul; 150(1):110-114.

11. Peeters ST, Heemsbergen WD, van Putten WL, Slot A, Tabak H, Mens JW et al. Acute and late complications after radiotherapy for prostate cancer: results of a multicenter randomized trial comparing 68 Gy to 78 Gy. Int J Radiat Oncol Biol Phys. 2005 Mar 15;61(4):1019-1034.

12. Emani B, Lyman J, Brown A, Coia L, Goitein M, Munzerider JE et al. Tolerance of normal tissue to therapeutic irradiation. Int J Radiat Oncol Biol Phys. 1991 May 15;21(1):109-122.

13. Lyman JT. Complication probability as assessed from dosevolume histograms. Radiat Res Suppl. 1985;8:S13-S19.

14. Kutcher GJ, Burman C. Calculation of complication probability factors for non-uniform normal tissue irradiation: the effective volume method. Int J Radiat Oncol Biol Phys. 1989 Jun; 16(6): 1623-1630.

15. Burman C, Kutcher GJ, Emami B, Goitein M. Fitting of normal tissue tolerance data to an analytic function. Int $\mathrm{J}$ Radiat Oncol Biol Phys. 1991 May 15;21(1):123-35.

16. Boehmer D, Bohsung J, Eichwurzel I, Moys A, Budach V. Clinical and physical quality assurance for intensity modulated radiotherapy of prostate cancer. Radiother Oncol. 2004 Jun; 71(3):319-325. Review.

17. Kuban D, Pollack A, Huang E, Levy L, Dong L, Starkschall $\mathrm{G}$ et al. Hazards of dose escalation in prostate cancer radiotherapy. Int J Radiat Oncol Biol Phys. 2003 Dec 1;57(5): 1260-1268.

18. Huang EH, Pollack A, Levy L, Starkschall G, Dong L, Rosen I et al. Late rectal toxicity: dose-volume effects of conformal radiotherapy for prostate cancer. Int $\mathrm{J}$ Radiat Oncol Biol Phys. 2002 Dec 1;54(5):1314-1321.

19. Fiorino C, Sanguineti G, Cozzarini C, Fellin G, Foppiano F, Menegotti L et al. Rectal dose-volume constraints in highdose radiotherapy of localized prostate cancer. Int $\mathrm{J}$ Radiat Oncol Biol Phys. 2003 Nov 15;57(4):953-962.

20. Bucciolini M, Buonamici FB, Casati M. Verification of IMRT fields by film dosimetry. Med Phys. 2004 Jan;31(1): 161-168.

21. Lawton CA, Won M, Pilepich MV, Asbell SO, Shipley WU, Hanks GE, et al. Long-term treatment sequelae following external beam irradiation for adenocarcinoma of the prostate: analysis of RTOG studies 7506 and 7706. Int J Radiat Oncol Biol Phys. 1991 Sep;21(4):935-939.

22. Hanks GE, Hanlon AL, Pinover WH, Horwitz EM, Schultheiss TE. Survival advantage for prostate cancer patients treated with high-dose three-dimensional conformal radiotherapy. Cancer J Sci Am. 1999 May-Jun;5(3): 152-158.

23. Zelefsky MJ, Fuks Z, Wolfe T, Kutcher GJ, Burman C, Ling $\mathrm{CC}$ et al. Locally advanced prostatic cancer: long-term toxicity outcome after three-dimensional conformal radiation therapy-a dose-escalation study. Radiology. 1998 Oct; 209(1):169-174. 
24. Zelefsky MJ, Fuks Z, Happersett L, Lee HJ, Ling CC, Burman $\mathrm{CM}$ et al. Clinical experience with intensity modulated radiation therapy (IMRT) in prostate cancer. Radiother Oncol. 2000 Jun;55(3):241-249.

25. Zelefsky MJ, Fuks Z, Hunt M, Yamada Y, Marion C, Ling $\mathrm{CC}$ et al. High-dose intensity modulated radiation therapy for prostate cancer: early toxicity and biochemical outcome in 772 patients. Int $J$ Radiat Oncol Biol Phys. 2002 Aug 1;53(5):1111-1116.

26. De Meerleer G, Vakaet L, Meersschout S, Villeirs G, Verbaeys $\mathrm{A}$, Oosterlinck $\mathrm{W}$, et al. Intensity-modulated radiotherapy as primary treatment for prostate cancer: acute toxicity in 114 patients. Int $J$ Radiat Oncol Biol Phys. 2004 Nov 1;60(3):777-787. Review.
27. Kupelian PA, Reddy CA, Carlson TP, Altsman KA, Willoughby TR. Preliminary observations on biochemical relapse-free survival rates after short-course intensitymodulated radiotherapy (70 Gy at 2.5 Gy/fraction) for localized prostate cancer. Int $J$ Radiat Oncol Biol Phys. 2002 Jul 15;53(4):904-912.

28. Zelefsky MJ, Chan H, Hunt M, Yamada Y, Shippy AM, Amols H. Long-term outcome of high dose intensity modulated radiation therapy for patients with clinically localized prostate cancer. J Urol. 2006 Oct;176(4 Pt 1): 1415-1419.

Correspondencia autor: Dr. L. Livi

Departamento de Radioterapia.

Universidad de Florencia. Italia

Información artículo: Original 